Pacific Journal of Mathematics

RINGS IN WHICH EVERY RIGHT IDEAL IS

SA AD H. MoHAM D AND SUR 


\section{RINGS IN WHICH EVERY RIGHT IDEAL IS QUASI-INJECTIVE}

\section{S. K. Jain, S. H. Mohamed and Surjeet Singh}

It is well known that if every right ideal of a ring $R$ is injective, then $R$ is semi simple Artinian. The object of this paper is to initiate the study of a class of rings in which each right ideal is quasi-injective. Such rings will be called $q$-rings. It is shown by an example that a $q$-ring need not be even semi prime. A number of important properties of $q$ rings are obtained.

Throughout this paper, unless otherwise stated, we assume that every ring has unity $1 \neq 0$. If $M$ is a right $R$-module, then $\hat{M}$ will denote the injective hull of $M$. For any positive integer $n, R_{n}$ will denote the ring of all $n \times n$ matrices over the ring $R . R^{4}, J(R)$ and $B(R)$ will denote the right singular ideal, the Jacobson radical and the prime radical respectively. A ring $R$ is said to be a right duo ring if every right ideal of $R$ is two-sided. Left duo rings are defined symmetrically. By a duo ring we mean a ring which is both right and left duo ring.

It is shown that $R_{n}(n>1)$ is a $q$-ring if and only if $R$ is semisimple Artinian. Some of the main results are: (i) a prime $q$-ring is simple Artinian, (ii) a semi-prime $q$-ring is a direct sum of two rings $S$ and $T$, where $S$ is a complete direct sum of simple Artinian rings, and $T$ is a semi-prime $q$-ring with zero socle, and (iii) a semi-prime $q$-ring is a direct sum of two rings $A$ and $B$, where $A$ is a right self injective duo ring, and $B$ is semi-simple artinian.

2. Let $R$ be a right self injective ring. If $B$ is any right ideal of $R$, then $\hat{B}=e R$ for some idempotent $e$ of $R$. Let $K=\operatorname{Hom}_{R}(\hat{B}, \hat{B})$. Then $K \cong e R e$. In fact every element in $K$ can be realized by the left multiplication of some element of eRe. By Johnson and Wong ([3], Theorem 1.1) $B$ is a quasi injective as a right $R$-module if and only if $K B=B$. Hence $B$ is quasi injective if and only if $B=K B=$ $(e R e) B=(e R)(e B)=\hat{B} B$. Hence every two-sided ideal in a right self injective ring is quasi-injective. So, the following is immediate.

2.1. Every commutative self injective ring is a $q$-ring.

Now, we give an example of a $q$-ring which is not semi-prime.

ExAmple 2.2. Let $Z$ be the ring of integers. Set $R=Z /(4)$. It is trivial that $R$ is a $q$-ring. But $R$ is not semi-prime, since its only proper ideal is nilpotent. 
In fact, $Z /(n)$ is a $q$-ring for every integer $n>1$, since it is self injective (cf. Levy [5]). Also we remark that $Z /(n)$ has nonzero nilpotent ideals if $n$ is not square free.

Next we prove

\section{THEOREM 2.3. The following are equivalent}

(1) $R$ is a q-ring

(2) $R$ is right self injective, and every right ideal of $R$ is of the form eI, $e$ is an idempotent in $R, I$ is a two sided ideal in $R$.

(3) $R$ is right self-injective, and every large right ideal of $R$ is two sided.

Proof. Assume (1). Therefore $R$ is right self injective. Let $B$ be any right ideal of $R$. Then $\hat{B}=e R$ for some idempotent $e$. Since $B$ is quasi injective $B=\hat{B} B=e R B=e I$, where $I=R B$, the smallest two-sided ideal of $R$ containing $B$. Hence (1) implies (2).

Assume (2). Let $A$ be a large right ideal of $R$. Then $A=e I$, $e^{2}=e, I$ is a two sided ideal. Since $A \cap(1-e) R=0,(1-e) R=0$. This implies that $e=1$. Hence $A=I$, proving (3).

Now assume (3). Let $B$ be a right ideal of $R$. If $K$ is a complement of $B$, then $B \oplus K$ is large in $R$. By assumption $B \oplus K$ is a two-sided ideal in $R$, hence quasi-injective. This implies $B$ is a quasi-injective, completing the proof.

TheORem 2.4. Let $n>1$ be an integer. Then $R_{n}$ is a q-ring if and only if $R$ is semi-simple Artinian.

Proof. Suppose that $R$ is not semi-simple Artinian. By Lambek ([4], Proposition 2, p. 61), there exists a large right ideal $B$ of $R$ such that $B \neq R$. Let $e_{i j}, 1 \leqq i, j \leqq n$ be the matrix units of $R_{n}$ and let $E=\left\{\sum a_{i j} e_{i j}: a_{1 j} \in B, 1 \leqq j \leqq n\right.$ and $\left.a_{i j} \in R, 1 \leqq i, j \leqq n\right\}$. It is clear that $E$ is a right ideal in $R_{n}$. But $E$ is not two-sided, for $e_{n n} \in E$ and $e_{1 n} e_{n n}=e_{1 n} \notin E$. Now, we prove that $E$ is a large right ideal in $R_{n}$. Let $0 \neq x=\sum_{i, j=1}^{n} b_{i j} e_{i j}$. If $b_{1 j}=0,1 \leqq j \leqq n$, then $x \in E$. So, let $b_{1 k} \neq 0$ for some $k$. Since $B$ is large in $R$, there exists $a \in R$ such that $0 \neq b_{1 k} a \in B$. Then,

$$
x\left(\alpha e_{k k}\right)=\left(\sum_{i, j=1}^{n} b_{i j} e_{i j}\right)\left(a e_{k k}\right)=\sum_{i=1}^{n} b_{i k} \alpha e_{i k} \in E .
$$

Hence, $0 \neq x\left(a e_{k k}\right) \in E$. Therefore $E$ is a large right ideal in $R_{n}$ which is not two-sided, and by Theorem $2.3, R_{n}$ is not a $q$-ring. This proves "only if" part. Other part is obvious.

We are now ready to show the existence of right self injective rings which are not $q$-rings. 
EXAMPLE 2.5. Let $R$ be a right self injective ring which is not semi-simple (we can take $R=Z /(4)$ ). Let $n>1$ be an integer. By Utumi ([6], Th. 8.3) $R_{n}$ is right self injective. But $R_{n}$ is not a $q$-ring, by the above theorem.

Next we prove

TheOREM 2.6. A simple ring is a q-ring if and only if it is Artinian.

Proof. Let $R$ be a simple $q$-ring. Let $B$ be a large right ideal in $R$. Then $B$ is two-sided, and hence $B=R$. This proves that $R$ does not contain any proper large right ideal. Hence $R$ is Artinian. The converse is trivial.

Now, we give an example of a right self injective simple ring which is not a $q$-ring.

EXAMPLE 2.7. Let $S$ be a noncommutative integral domain which is not a right Öre domain (cf. Goldie [1]). Let $R=\hat{S}$. Then $R$ is a right self injective simple regular ring which is not Artinian. By the above theorem $R$ is not a $q$-ring.

Lemma 2.8. Let $R$ be a q-ring. Then $B(R)$ is essential in $J(R)$ as a right $R$-module.

Proof. Since $R$ is self injective, $J(R)=R^{4}$, by Utumi ([6], Lemma 4.1). Let $0 \neq x \in J(R)$. There exist a large right ideal $E$ of $R$ such that $x E=0$. Then $x E \subset P$ for every prime ideal $P$ of $R$. Since $R$ is a $q$-ring, $E$ is two-sided. This implies that either $x \in P$ or $E \subset P$.

Let $\left\{P_{i}\right\}_{i \in I}$ be the set of all prime ideals of $R$ such that $x \in P_{i}$ for every $i \in I$, and $\left\{P_{j}\right\}_{j \in J}$ be the set of all prime ideals of $R$ such that $x \notin P_{j}$ for every $j \in J$. Let $X=\bigcap_{i \in I} P_{i}$, and $Y=\bigcap_{j \in J} P_{j}$. $X \neq 0$, since $0 \neq x \in X$. On the other hand, $E \subset P_{j}$ for every $j \in J$. Thus $E \subset Y$, which implies that $Y$ is large in $R$. Therefore $B(R)=$ $X \cap Y \neq(0)$. Moreover, there exists $\mathrm{a} \in R$ such that $0 \neq x a \in Y$. This implies that $0 \neq x a \in X \cap Y=B(R)$, completing the proof.

Hence, we have the following

THEOREM 2.9. A q-ring is regular if and only if it is semiprime. 
Proof. The result follows by the above lemma, and Utumi ([6], Corollary 4.2).

THEOREM 2.10. Let $V$ be a vector space over a division ring $D$, and let $R=\operatorname{Hom}_{D}(V, V)$. Then $R$ is a q-ring if and only if $V$ is of finite dimension over $D$.

Proof. The "if" part is obvious. Conversely, suppose that $V$ is of infinite dimension over $D$. Let $X=\left\{x_{1}, x_{2}, \cdots\right\}$ be a denumerable set of linearly independent elements of $V . X$ can be extended to a basis $X \cup Y$ of $V$. Let $F$ be the ideal in $R$ consisting of all elements of finite rank. Let $\sigma \in R$ be defined by $\sigma\left(x_{2 i}\right)=x_{2 i}, \sigma\left(x_{2 i-1}\right)=0$ for every $i$, and $\sigma(y)=0$ for every $y \in Y$. Let $E=\sigma R+F$. Then $F \subset E$. Since $F$ is a two-sided ideal in $R, F$ is large. Therefore $E$ is a large right ideal in $R$. We proceed to prove that $E$ is not twosided. Let $\lambda_{1}, \lambda_{2} \in R$ be defined by: $\lambda_{1}\left(x_{i}\right)=x_{2 i}$ for every $i$, and $\lambda_{1}(y)=$ 0 for every $y \in Y, \lambda_{2}\left(x_{2 i}\right)=x_{i}, \lambda_{2}\left(x_{2 i-1}\right)=0$, for every $i$, and $\lambda_{2}(y)=0$ for every $y \in Y$. Let $\lambda=\lambda_{2} \sigma \lambda_{1}$. Then $\lambda\left(x_{i}\right)=x_{i}$ for every $i$. Hence $X \subset \lambda(V)$. We assert that $\lambda \notin E$; for otherwise, let $\lambda=\sigma r+f, r \in R$, $f \in F$. Then $X \subset \lambda(V)=(\sigma r+f)(V) \subset \sigma(V)+f(V)$. But since $f$ is of finite rank, there exists an integer $n$ such that $x_{2 n-1} \notin f(V)$. Also, by definition of $\sigma, x_{2 n-1} \notin \sigma(V)$. Hence $x_{2 n-1} \notin \sigma(V)+f(V)$, which is a contradiction. Thus $\lambda \notin E$, as desired. However $\lambda \in R \sigma R \subset R E$. Hence $E$ is not a two-sided ideal. Therefore, by Theorem 2.3, $R$ is not a $q$-ring. This completes the proof.

We remark that the above theorem is also a consequence of Theorem 2.4.

The right (left) socle of a ring $R$ is defined to be the sum of all minimal right (left) ideals of $R$. It is well known that in a semiprime ring $R$, the right and left socles of $R$ coincide, and we denote any of them by soc $R$.

LEMMA 2.11. A semi-prime q-ring $R$ with zero socle is strongly regular.

Proof. Let $M$ be a maximal right ideal in $R$. Either $M$ is a direct summand of $R$ or $M$ is large in $R$. If $M$ is a direct summand of $R$, then its complement is a minimal right ideal. This implies that soc $R \neq 0$, a contradiction. Therefore, every maximal right ideal is large, hence two-sided. By Lemma 2.8, $J(R)=0$. Thus $R$ is isomorphic to a subdirect sum of division rings, which implies that $R$ has no nonzero nilpotent elements. Since $R$ is regular, by Theorem $2.9, R$ is strongly regular. 
Lemma 2.12. A prime q-ring has nonzero socle.

Proof. Let $R$ be a prime $q$-ring. If possible, let soc $R=0$. By the above lemma, $R$ is strongly regular. Hence $R$ is a division ring, and $\operatorname{soc} R=R$ contradicting our assumption. Therefore soc $R \neq(0)$.

THEOREM 2.13. A prime ring $R$ is a q-ring if and only if $R$ is simple Artinian.

Proof. By Theorem 2.9, and the above lemma, $R$ is a prime regular ring with nonzero socle. Hence, by Johnson ([2], Th. 3.1), $\hat{R}=\operatorname{Hom}_{D}(V, V)$, where $V$ is some vector space over a division ring $D$. But then $R=\operatorname{Hom}_{D}(V, V)$, since $R$ is right self injective. By Theorem 2.10, $V$ has finite dimension over $D$. Let $(V: D)=n$. Then $R \cong D_{n}$, completing the proof.

Lemma 2.14. Let $\left\{R_{\alpha}\right\}_{\alpha \in I}$ be a finite set of rings. Then the direct sum $\sum_{\alpha \in I} \oplus R_{\alpha}$ is a q-ring if and only if each $R_{\alpha}$ is a q-ring.

The proof is obvious.

That Lemma 2.14 is not true for an infinite number of rings is shown by the following example which is due to Storrer.

EXAMPLE 2.15. Let $R$ be a $2 \times 2$-matrix ring over a field $F$. Let $\left\{R_{\alpha}\right\}_{\alpha \in I}$ be an infinite family of copies of $R$ and let $S=\pi R_{\alpha}, \alpha \in I$. Let $E$ be the right ideal of $S$ consisting of those elements $\left[x_{\alpha}\right]$ of $S$ such that all but finite $x_{\alpha}^{\prime} \mathrm{s}$ are matrices with first row zero. Since $R_{\alpha} \subset E$ for all $\alpha \in I, E$ is a large right ideal of $S$. To show that $E$ is not two-sided, consider $\left[x_{\alpha}\right] \in E$ where $x_{\alpha}=\left(\begin{array}{ll}0 & 0 \\ 1 & 0\end{array}\right)$ for all $\alpha \in I$. Let $\left[y_{\alpha}\right] \in S$ be such that $y_{\alpha}=\left(\begin{array}{ll}0 & 1 \\ 0 & 0\end{array}\right)$ for all $\alpha \in I$. Then $\left[y_{\alpha}\right]\left[x_{\alpha}\right]=$ $\left[z_{\alpha}\right]$, where $z_{\alpha}=\left(\begin{array}{ll}1 & 0 \\ 0 & 0\end{array}\right)$. But then $\left[z_{\alpha}\right] \notin E$, and $E$ is not two-sided. Hence, by Theorem 2.3, $S$ is not a $q$-ring.

Example 2.15 also suggests the following.

THEOREM 2.16. Let $\left\{R_{\alpha}\right\}_{\alpha \in I}$ be a family of simple Artinian rings and let $R$ be their complete direct sum. Then $R$ is a q-ring if and only if all $R_{\alpha}^{\prime} s$ excepting a finite number of them are division rings.

The above theorem shows, in particular, that a regular $q$-ring may not be Artinian.

LEMMA 2.17. Let $R$ be a semi-prime q-ring such that $\operatorname{soc} R$ is 
large in $R$. Then $R$ is a complete direct sum of simple Artinian rings.

Proof. Since soc $R$ is large, every nonzero right ideal of $R$ contains a minimal right ideal. Also $R$ is regular, by Theorem 2.9. Hence by Johnson ([2], Th. 3.1), $R$ is a complete direct sum of rings $R_{i}$, where each $R_{i}$ is the ring of all linear transformations of some vector space $V_{i}$;over a division ring $D_{i}$. But then by Lemma 2.14 and Theorem 2.10, each $R_{i}$ is a simple Artinian ring. This completes the proof.

In the following two theorems we assume that every ring has a unity element which may be equal to zero.

THEOREM 2.18. Let $R$ be a semi-prime q-ring. Then $R=S \oplus T$, where $S$ is a complete direct sum of simple Artinian rings and $T$ is a semi-prime q-ring with zero socle.

Proof. Let $F=\operatorname{soc} R$. Since $R^{\Delta}=0, \hat{F}=\{x \in R: x E \subset F$ for some large right ideal $E$ of $R$ \}. Then it is immediate that $\hat{F}$ is a two-sided ideal in $R$. Since $R$ is self injective, $\hat{F}=e R$ for some idempotent $e$. Then $e$ is central, since $R$ is regular. Let $S=e R$ and $T=(1-e) R$. Hence $R=S \oplus T$. By Lemma 2.14, both $S$ and $T$ are $q$-rings. Further, it can be easily verified that (i) $S$ is a semiprime ring, soc $S=F$, and $F$ is large in $S$, and (ii) $T$ is a semi-prime ring with zero socle. By the above lemma $S$ is a complete direct sum of simple Artinian rings, completing the proof.

As a consequence of Lemma 2.11, Theorem 2.16 and Theorem 2.18 we have the following.

THEOREM 2.19. A semi-prime ring $R$ is a q-ring if and only if $R=A \oplus B$, where $A$ is a right self injective duo ring and $B$ is semi-simple Artinian.

\section{REFERENCES}

1. A. W. Goldie, Semi prime rings with maximum condition, Proc. London Math. Soc. $10(1960)$

2. R. E. Johnson, Quotient rings of rings with zero singular ideal, Pacific J. Math. 11, (1961).

3. R. E. Johnson and E. T. Wong, Quasi injective module and irreducible rings, J. London Math. Soc. 36 (1961).

4. J. Lambek, Lectures on rings and modules. 
5. L. S. Levy, Commutative rings whose homomorphic images are self-injective, Pacific J. Math. 18 (1966).

6. Y. Utumi, On continuous rings and self injective rings, Trans. Amer. Math. Soc. 118 (1965).

Received March 5, 1969.

UNIVERSITY OF DELHI INDIA 



\section{PACIFIC JOURNAL OF MATHEMATICS}

\section{EDITORS}

H. ROYDEN

Stanford University

Stanford, California

Richard Pierce

University of Washington

Seattle, Washington 98105
J. DUGUNDJI

Department of Mathematics

University of Southern California

Los Angeles, California 90007

BASIL GORDON

University of California

Los Angeles, California 90024

\section{ASSOCIATE EDITORS}
E. F. BECKENBACH
B. H. NEUMANN
F. WOLF
K. YosHIDA

\section{SUPPORTING INSTITUTIONS}

UNIVERSITY OF BRITISH COLUMBIA

CALIFORNIA INSTITUTE OF TECHNOLOGY

UNIVERSITY OF CALIFORNIA

MONTANA STATE UNIVERSITY

UNIVERSITY OF NEVADA

NEW MEXICO STATE UNIVERSITY

OREGON STATE UNIVERSITY

UNIVERSITY OF OREGON

OSAKA UNIVERSITY

UNIVERSITY OF SOUTHERN CALIFORNIA
STANFORD UNIVERSITY

UNIVERSITY OF TOKYO

UNIVERSITY OF UTAH

WASHINGTON STATE UNIVERSITY

UNIVERSITY OF WASHINGTON

*

AMERICAN MATHEMATICAL SOCIETY CHEVRON RESEARCH CORPORATION TRW SYSTEMS

NAVAL WEAPONS CENTER 


\section{Pacific Journal of Mathematics}

\section{Vol. 31, No. $1 \quad$ November, 1969}

James Burton Ax, Injective endomorphisms of varieties and schemes........

Richard Hindman Bouldin, A generalization of the Weinstein-Aronszajn

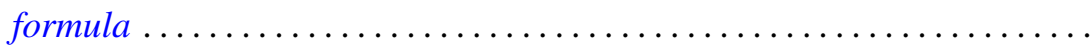

John Martin Chadam, The asymptotic behavior of the Klein-Gordon equation with external potential. II ...............................

Rina Hadass, On the zeros of the solutions of the differential equation

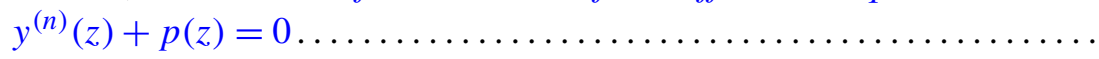

John Sollion Hsia, Integral equivalence of vectors over local modular lattices. II .............................................

Robert Hughes, Boundary behavior of random valued heat polynomial

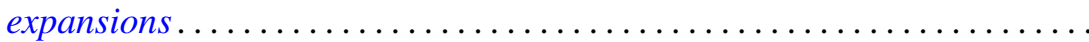

Surender Kumar Jain, Saad H. Mohamed and Surjeet Singh, Rings in which every right ideal is quasi-injective .........................

T. Kawata, On the inversion formula for the characteristic function .........

Erwin Kleinfeld, On right alternative rings without proper right ideals......

Robert Leroy Kruse and David Thomas Price, On the subring structure of

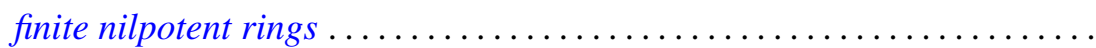

Marvin David Marcus and Stephen J. Pierce, Symmetric positive definite multilinear functionals with a given automorphism. .

William Schumacher Massey, Pontryagin squares in the Thom space of a bundle...

William Schumacher Massey, Proof of a conjecture of Whitney ...

John William Neuberger, Existence of a spectrum for nonlinear transformations

Stephen E. Newman, Measure algebras on idempotent semigroups ...

$\mathrm{K}$. Chandrasekhara Rao, Matrix transformations of some sequence spaces

Robert Bruce Schneider, Some theorems in Fourier analysis on symmetric

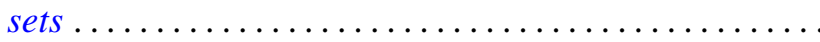

Ulrich F. K. Schoenwaelder, Centralizers of abelian, normal subgroups of hypercyclic groups...

Jerrold Norman Siegel, $G$-spaces, $H$-spaces and $W$-spaces

Robert Irving Soare, Cohesive sets and recursively enumerable Dedekind cuts...

Kwok-Wai Tam, Isometries of certain function spaces .... . .

Awadhesh Kumar Tiwary, Injective hulls of semi-simple modules over regular rings ....

Eldon Jon Vought, Concerning continua not separated by any nonaposyndetic subcontinuum .................... 